\title{
Sex and hedgehog: roles of genes in the hedgehog signaling pathway in mammalian sexual differentiation
}

\author{
Heather L. Franco • Humphrey H.-C. Yao
}

Published online: 22 November 2011

(C) Springer Science+Business Media B.V. (Outside the USA) 2011

\begin{abstract}
The chromosome status of the mammalian embryo initiates a multistage process of sexual development in which the bipotential reproductive system establishes itself as either male or female. These events are governed by intricate cell-cell and interorgan communication that is regulated by multiple signaling pathways. The hedgehog signaling pathway was originally identified for its key role in the development of Drosophila, but is now recognized as a critical developmental regulator in many species, including humans. In addition to its developmental roles, the hedgehog signaling pathway also modulates adult organ function, and misregulation of this pathway often leads to diseases, such as cancer. The hedgehog signaling pathway acts through its morphogenetic ligands that signal from ligandproducing cells to target cells over a specified distance. The target cells then respond in a graded manner based on the concentration of the ligands that they are exposed to. Through this unique mechanism of action, the hedgehog signaling pathway elicits cell fate determination, epithelial-mesenchymal interactions,
\end{abstract}

Responsible Editor: Tariq Ezaz and Jennifer Graves.

H. L. Franco • H. H.-C. Yao $(\bowtie)$

Reproductive Developmental Biology Group,

Laboratory of Reproductive and Developmental Toxicity,

National Institute of Environmental Health Sciences,

111 TW Alexander Drive, C4-10,

Research Triangle Park, NC 27709, USA

e-mail: humphrey.yao@nih.gov and cellular homeostasis. Here, we review current findings on the roles of hedgehog signaling in the sexually dimorphic development of the reproductive organs with an emphasis on mammals and comparative evidence in other species.

Keywords Hedgehog · testis · ovary · germ cells · reproductive tract $\cdot$ external genitalia $\cdot \operatorname{sex}$ determination

\begin{tabular}{|c|c|}
\hline \multicolumn{2}{|c|}{ Abbreviations } \\
\hline AMH & Anti-Müllerian hormone \\
\hline COUP-TFII & $\begin{array}{l}\text { Chicken ovalbumin upstream promoter } \\
\text { transcription factor II }\end{array}$ \\
\hline DHH & Desert hedgehog \\
\hline E & Embryonic day \\
\hline IHH & Indian hedgehog \\
\hline INSL3 & Insulin-like factor 3 \\
\hline PTCH & Patched \\
\hline SF1 & Steroidogenic factor 1 \\
\hline $\mathrm{SHH}$ & Sonic hedgehog \\
\hline SMO & Smoothened \\
\hline SRY & $\begin{array}{l}\text { Sex-determining gene of the Y } \\
\text { chromosome }\end{array}$ \\
\hline
\end{tabular}

\section{Introduction}

In mammals, sexually dimorphic establishment of the reproductive system follows three distinct steps. Initial sex determination (also known as chromosomal 
sex determination) occurs at the time of fertilization when sperm carrying either a $\mathrm{Y}$ or $\mathrm{X}$ chromosome fuses with the $\mathrm{X}$ chromosomebearing oocyte (Welshons and Russell 1959). Once sex chromosome composition of the embryos is established (XY vs. XX), the second step (primary or gonadal sex determination) proceeds, in which the gonadal structure (testis or ovary) is specified. The sex-determining gene on the $\mathrm{Y}$ chromosome $(S R Y)$ is both necessary and sufficient to initiate testis morphogenesis (Koopman et al. 1991; Lovell-Badge and Robertson 1990). On the other hand, in the XX embryo, the absence of the SRY gene results in the initial formation of the ovary. Following the establishment of the gonad, the final stage of sex differentiation (or secondary sex determination) occurs when sex-specific development of the reproductive tracts and external genitalia are completed. Initially, the Wolffian and Müllerian ducts, the precursors of the male and female reproductive tracts, respectively, develop in both male and female embryos (Kobayashi and Behringer 2003). In male embryos, the testes produce anti-Müllerian hormone $(\mathrm{AMH})$ and testosterone, which cause Müllerian duct regression and differentiation of the Wolffian duct into the epididymis, vas deferens, and seminal vesicles, respectively. Female embryos do not produce these hormones, and therefore, the Wolffian duct regresses and the Müllerian duct is maintained and forms the oviduct, uterus, cervix, and upper part of the vagina. Androgens produced by the testes also masculinize the male external genitalia, and the absence of androgens in female leads to the development of female genitalia. This three-step sexual differentiation process is clearly initiated by sex chromosomes and its components. However, downstream morphogenetic events, such as the appearance of gonad-specific cell types and structure, reproductive tract differentiation, and external genitalia establishment, require intricate interactions between systemic endocrine and local signaling pathways. One locally regulated signaling pathway that has emerged as a key regulator of mammalian sexual differentiation is the hedgehog signaling pathway.

The hedgehog signaling pathway was originally identified in a Drosophila screen and was found to be essential for larval body patterning in flies (NussleinVolhard and Wieschaus 1980). Since its discovery, the hedgehog signaling pathway has been implicated in organogenesis of multiple species from worms to humans. In addition, aberrant regulation of the hedgehog signaling pathway is linked to numerous diseases in humans, such as basal cell carcinoma, medulloblastoma, rhabdomyosarcoma, and glioma (Varjosalo and Taipale 2008). The hedgehog signaling pathway is activated by hedgehog ligands, which act as morphogens as they travel over both short and long distances to their target cells where their effect is exerted (Wilson and Chuang 2010). In most tissues, the hedgehog signaling pathway mediates epithelialmesenchymal communication, a critical process for tissue remodeling and differentiation. Because the molecular aspects of the hedgehog signaling pathway in different species have been extensively reviewed elsewhere (Driver et al. 2008; Turner et al. 2004; Varjosalo and Taipale 2008), only a brief description of its mechanism of action in mammals is provided below. Three mammalian hedgehog ligands have been identified: desert hedgehog (DHH), Indian hedgehog (IHH), and sonic hedgehog (SHH) (Varjosalo and Taipale 2008). In the absence of ligands, the hedgehog membrane receptor patched (PTCH1 or PTCH2) represses the action of another membrane-bound protein called smoothened (SMO). Upon ligand binding to patched, its repression of SMO is relieved, leading to activation of SMO and its downstream intracellular signaling cascade. SMO activation induces the processing of the GLI-Krüppel family of transcription factors (GLI1, GLI2, and GLI3), which results in their transcriptional activation and translocation to the nucleus, where they regulate a number of hedgehog target genes, including the induction of Ptch1 and Gli1. Analysis of the evolutionary history of the hedgehog signaling pathway reveals that the core components of this pathway are conserved throughout metazoans (Ingham et al. 2011; Wilson and Chuang 2010). Genes that encode the hedgehog components are located on the autosomes and are well conserved from flies through mice, rats, cows, and humans (Table 1). Interestingly, Caenorhabditis elegans also express hedgehog signaling components, such as Patched and Gli, although no ortholog of the hedgehog ligand has been identified in this species.

To better understand how sexual differentiation and reproductive organ formation are accomplished, it is important to identify the players that facilitate cell-cell 
Table 1 Chromosome localization of the core components of the hedgehog signaling pathway in various species

\begin{tabular}{|c|c|c|c|c|c|c|c|}
\hline \multirow[t]{2}{*}{ Gene } & \multicolumn{7}{|l|}{ Species } \\
\hline & $\begin{array}{l}\text { C. elegans } \\
\text { (worm) }\end{array}$ & $\begin{array}{l}\text { D. melanogaster } \\
\text { (fruitfly) }\end{array}$ & $\begin{array}{l}\text { D. ranio } \\
\text { (zebrafish) }\end{array}$ & $\begin{array}{l}\text { M. musculus } \\
\text { (mouse) }\end{array}$ & $\begin{array}{l}R . \text { norvegicus } \\
\text { (rat) }\end{array}$ & $\begin{array}{l}\text { B. taurus } \\
\text { (cow) }\end{array}$ & $\begin{array}{l}\text { H. sapiens } \\
\text { (human) }\end{array}$ \\
\hline Desert Hedgehog & $\mathrm{n} / \mathrm{a}$ & 3(hh) & 23 & 15 & 7 & 5 & 12 \\
\hline Indian Hedgehog & $\mathrm{n} / \mathrm{a}$ & & 9 (ihha), 6 (ihhb) & 1 & 9 & 2 & 2 \\
\hline Sonic Hedgehog & $\mathrm{n} / \mathrm{a}$ & & 7 (shha), 2 (shhb) & 5 & 4 & 4 & 7 \\
\hline Patched1 & 11 (ptc-1) & 2 (ptc) & $8(\mathrm{ptc} 1)$ & 13 & 17 & 8 & 9 \\
\hline Patched2 & $11($ ptc-2) & & $2(\operatorname{ptc} 2)$ & 4 & 5 & 3 & 1 \\
\hline Smoothened & $\mathrm{n} / \mathrm{a}$ & 2 & 4 & 6 & 4 & 4 & 7 \\
\hline Gli1 & $3($ tra- -1$)$ & 4(ci) & 1 & 10 & 7 & 5 & 12 \\
\hline Gli2 & $\mathrm{n} / \mathrm{a}$ & & 9 (gli2a), 11 (gli2b) & 1 & 13 & 2 & 2 \\
\hline Gli3 & $\mathrm{n} / \mathrm{a}$ & & 24 & 13 & 17 & 4 & 7 \\
\hline
\end{tabular}

communication, cell fate determination, and tissue remodeling in these processes. Components of the hedgehog signaling pathway are expressed in the male and female reproductive systems in distinct spatial and temporal patterns (Walterhouse et al. 2003). Here, we provide a historical and comparative review on the involvement of the hedgehog signaling pathway in the sexual differentiation of the mammalian embryo (mostly in mice) as well as other species.

\section{The original frontier of hedgehog signaling in mammalian reproduction}

The first indication of a role for hedgehog signaling in sexual differentiation came from studies exploring the action of DHH in the mouse embryo. Within the fetal testis, Dhh is expressed specifically in the Sertoli cells as early as embryonic day or E11.5, whereas the other two ligands are not produced (Bitgood and McMahon 1995). Both Ptch1 and Gli1 are present in the interstitium of the testis, specifically in the Leydig and peritubular myoid cells, indicating that the hedgehog signaling pathway participates in epithelialmesenchymal communication within the fetal testis (Barsoum and Yao 2011; Bitgood et al. 1996; Yao et al. 2002). Ablation of Dhh resulted in testis phenotypes that differed depending upon the genetic background of the mouse (Bitgood et al. 1996; Clark et al. 2000; Pierucci-Alves et al. 2001; Yao et al. 2002). On a pure $129 / \mathrm{Sv}$ background, $D h h^{-/-}$mice displayed an adult testis phenotype characterized by a spermatogenesis defect (Bitgood et al. 1996). However, when $D h h^{-/-}$mice were generated on a mixed background (129/Sv, C57BL/6, and Swiss Webster), the phenotypes became exacerbated, displaying fetal testis defects including disorganized testis cords as well as Leydig and peritubular myoid cell defects (Clark et al. 2000; Pierucci-Alves et al. 2001; Yao et al. 2002). These strain-specific effects of DHH demonstrate the importance of DHH in germline and somatic cell development in fetal life and adulthood as well as the possible involvement of genetic modifiers that modulate DHH activity.

Hedgehog signaling influences germ cell development

The initial finding of the spermatogenesis defect in the $D h h^{-/-}$mice was puzzling as downstream hedgehog signaling components, such as Ptch1, Gli1, and Gli2, are expressed exclusively in the testis interstitium and are absent in male germ cells in the fetal testes (Barsoum and Yao 2011; Bitgood and McMahon 1995; Bitgood et al. 1996; Yao et al. 2002). This leads to the possibility that $D h h$ indirectly influences spermatogenesis by acting on the interstitial cell population. However, it was later discovered that male germ cells in the adult testis could be a direct target of hedgehog signaling based on the presence of downstream components, such as Gli1, Gli2, Gli3, Ptch1, Ptch2, Ptchd3, and Smo, in the spermatogenic 
cell populations (Fan et al. 2007; Kroft et al. 2001; Morales et al. 2009; Szczepny et al. 2006). Similar to the $D h h^{-/-}$model, ectopic activation of the hedgehog signaling pathway by overexpression of Gli1 led to a block in spermatogenesis at the primary spermatocyte stage (Kroft et al. 2001). These findings highlight the importance of a properly tuned hedgehog signaling pathway in spermatogenesis.

The hedgehog signaling pathway also plays a role in germ cell development in species other than mice. In Drosophila, the primordial germ cells form a 16-cell cyst at which point they differentiate to form either the spermatocyte in males or the oocyte and supporting nurse cells in the female (Jemc 2011). Evidence of an influence of the hedgehog signaling pathway on Drosophila spermatogenesis has not been demonstrated; however, the hedgehog signaling pathway could be involved in sex determination of the germ cells. The autosomal gene Sex-lethal, which controls somatic cell sex determination and female germ cell differentiation, is a target of the hedgehog signaling pathway in the germline (Vied and Horabin 2001). The hedgehog signaling pathway also influences female germ cell proliferation and the eventual specification of the oocyte from within the 16-cell cyst (Sato et al. 2010; Xia et al. 2010). In C. elegans, mutation of the ptc-1 gene, the homolog of Ptch, led to failed cytokinesis of germ cells and subsequent infertility in both males and females (Kuwabara et al. 2000). While the development of germ cells in C. elegans is interesting, it is equally striking how hermaphrodites make the decision to produce sperm or oocytes. This process is largely unclear; however, it was recently demonstrated that the MPK-1/ERK signaling pathway initiates a series of events including degradation of tra-1 to promote oogenesis (Arur et al. 2011). Thus, the Hedgehog signaling pathway may act within female germ cells to promote their specification and proliferation. It remains to be seen if the hedgehog signaling pathway also acts in this capacity in the mammalian oocyte.

In species such as fruit flies and zebrafish, the hedgehog signaling pathway was initially implicated in the migration of primordial germ cells. In Drosophila, primordial germ cells arise from a deposit of maternal cytoplasm called germ plasm in the posterior portion of the embryo, and during invagination, they migrate to the mesoderm where they intermix with the somatic precursor cells (Jemc 2011). The hedgehog signaling pathway was initially proposed to be necessary for germ cell migration as an attractive cue for their migration (Deshpande et al. 2001). However, these findings were recently disputed (Renault et al. 2009). In zebrafish embryos, treatment with the hedgehog inhibitor cyclopamine inhibited germ cell migration as a result of a systemic defect in cell motility (Mich et al. 2009). However, zebrafish with mutations in the hedgehog signaling pathway (Shh, Gli1, Gli2a, and Smo) failed to display this phenotype. In fact, it was found that cyclopamine affected cell adhesion in zebrafish, which then impact the motility of the primordial germ cells. Therefore, it appears as though the hedgehog signaling pathway is not required for germ cell migration, at least in zebrafish. The involvement of the hedgehog signaling pathway in germ cell migration in mammals remains to be determined.

Hedgehog signaling is essential for fetal Leydig cell development

The most well studied cell type that the hedgehog signaling pathway targets is the fetal Leydig cell. Fetal Leydig cells, the steroidogenic cells of the testis, produce androgens and insulin-like factor 3 (INSL3), which are responsible for the maintenance and differentiation of the male reproductive tract, the development of the external genitalia, and testis descent (Barsoum and Yao 2009). As mentioned above, ablation of Dhh in mice on a mixed background led to a reduction in the number of fetal Leydig cells (Clark et al. 2000; Pierucci-Alves et al. 2001; Yao et al. 2002). This regulation requires an interaction with the orphan nuclear receptor steroidogenic factor 1 (SF1) as loss of one copy of $S f 1$ exacerbates the Leydig cell defect of the $D h h^{-1-}$ mice (Park et al. 2007). A role for DHH in Leydig cell differentiation appears conserved as rats that contain a spontaneous mutation in $\mathrm{DHH}$ exhibit Leydig cell phenotypes similar to those of the Dhh knockout mice (Kawai et al. 2011). Interestingly, in in vitro organ culture experiments, treatment with a general hedgehog inhibitor, cyclopamine, completely blocked Leydig cell development, implying that hedgehog ligands, in addition to $\mathrm{DHH}$, could be involved in Leydig cell development (Yao and Capel 2002). SHH, produced by 
the neighboring mesonephros, could be the additional source of hedgehog ligand. Mesonephros-derived SHH is apparently active based on the positive expression of Ptch1 and Gli1 in this region (Barsoum and Yao 2011; Bitgood et al. 1996; Yao et al. 2002) (Bitgood and McMahon 1995). Interestingly, Ptch1 and Glil expression in the mesonephros is not affected by the loss of Dhh, further supporting a potential role for SHH (Bitgood et al. 1996). In a recent report examining the importance of posttranslational modifications of SF1, ectopic expression of Shh and upregulation of other hedgehog components were found in the fetal testis interstitium when SF1 sumoylation was eliminated (Lee et al. 2011). The testis of the mutant mice displayed altered testis cord morphology, which was accompanied by an increase in Leydig cell function. Intriguingly, expansion of Shh expression was also found in the fetal adrenal of the mutant mice where testis specific markers, such as $\operatorname{Sox} 9$, were induced. These observations not only provide a novel mechanism for SF1 in the transcriptional regulation of $\mathrm{Sh} h$ but also demonstrate the ability of SHH to influence testis development.

Further evidence of hedgehog regulation of the Leydig cell population came from studies examining the downstream effectors of the hedgehog signaling pathway in this process. The ectopic activation of SMO in the fetal ovary, where the hedgehog signaling pathway is normally inactive, promotes the ectopic appearance of Leydig cells (Barsoum et al. 2009). The actions of SMO are mediated through the Gli family of transcriptional activators, including Gli1 and Gli2. Overexpression of Gli1 reportedly resulted in Leydig cell hyperplasia although no specific Leydig cell markers were examined (Kroft et al. 2001). Ablation of either Gli1 or Gli2 does not affect Leydig cell development; however, treatment with the Gli inhibitor GANT61 inhibits Leydig cell formation (Barsoum and Yao 2011). A potential hedgehog downstream effector in Leydig cell development is chicken ovalbumin upstream promoter transcription factor II (COUP-TFII) (Qin et al. 2008). COUP-TFII is an orphan nuclear receptor implicated in the hedgehog signaling pathway in multiple tissues (Lin et al. 2011). When it was ablated in the adult mouse, both testis and epididymal weights were reduced (Qin et al. 2008). The testis displayed a reduced number of Leydig cells, which led to the reduced epididymal weight as androgen treatment was able to rescue the epididymal phenotype. It remains to be determined whether COUP-TFII is indeed a downstream regulator of the hedgehog signaling pathway in fetal Leydig cells. Together, these data point to a role for the hedgehog signaling pathway and its downstream components in the regulation of the fetal Leydig cell population.

Proper testis cord formation in the fetal testis requires hedgehog signaling

Disruption of the hedgehog signaling pathway, either genetically or pharmacologically, resulted in defects in testis cord formation (Clark et al. 2000; PierucciAlves et al. 2001; Szczepny et al. 2008; Yao and Capel 2002; Yao et al. 2002). The hedgehog signaling pathway is active in the testis interstitium, which contains fetal Leydig cells, peritubular myoid cells, and vasculature (Barsoum and Yao 2011; Bitgood et al. 1996; Yao et al. 2002). Peritubular myoid cells are critical for testis cord formation as they, along with Sertoli cells, secrete components of the basal lamina that delineate the testis cords (Tung et al. 1984). Ablation of $D h h$ in mice on a mixed genetic background resulted in peritubular myoid cell defects characterized by focal disruption of the basal lamina (Clark et al. 2000; Pierucci-Alves et al. 2001). However, little is known about the mode of action of the hedgehog signaling pathway in the peritubular myoid cell due to the lack of specific markers for them (Jeanes et al. 2005). One of the critical events in testis cord formation is the migration of endothelial cells from the mesonephros and the establishment of the testis vascular network (Combes et al. 2009; Cool et al. 2011). The vasculature within the testis has not been demonstrated to express hedgehog signaling components (Barsoum and Yao 2011; Bitgood et al. 1996; Yao et al. 2002). Furthermore, loss of hedgehog signaling, either by ablation of $D h h$ or treatment with cyclopamine, does not impact cell migration from the mesonephros or vascular formation (Bitgood et al. 1996; Clark et al. 2000; Pierucci-Alves et al. 2001; Yao and Capel 2002; Yao et al. 2002). Therefore, it does not appear as though the hedgehog signaling pathway acts upon the testis vascular network.

The temporal and cell-type-specific expression pattern of Dhh (right after the onset of testis determination in Sertoli cells) and its functions has 
made DHH the first identified morphogenetic regulator downstream of the testis-determining switch SRY. Sertoli cell-derived DHH facilitates testis cord formation by acting upon peritubular myoid cells and, at the same time, inducing fetal Leydig cell differentiation (Fig. 1). How DHH induces distinct cellular responses in these two cell populations is not clear. Precursors of peritubular myoid cells and fetal Leydig cells are thought to derive from two separate populations (SF1 negative for peritubular myoid cells and SF1 positive for Leydig cells). The presence or absence of SF1 could contribute to the distinct responses to $\mathrm{DHH}$ of these two cell types (Fig. 1). This hypothesis is supported by the observation that when only one functional $S f 1$ allele is present in the $D h h^{-/-}$male, Leydig cell defects are exacerbated, whereas the testis cord malformation was not affected (Park et al. 2007). Future studies aimed at determining how the $\mathrm{DHH}$ response differs in these two cell types should shed light on the involvement of SF1 in this process.

\section{Does the hedgehog signaling pathway play a role in the female gonad?}

The hedgehog signaling pathway is inactive in the fetal ovary based on the absence of Ptch1 and Gli1 expression (Barsoum and Yao 2011; Bitgood and

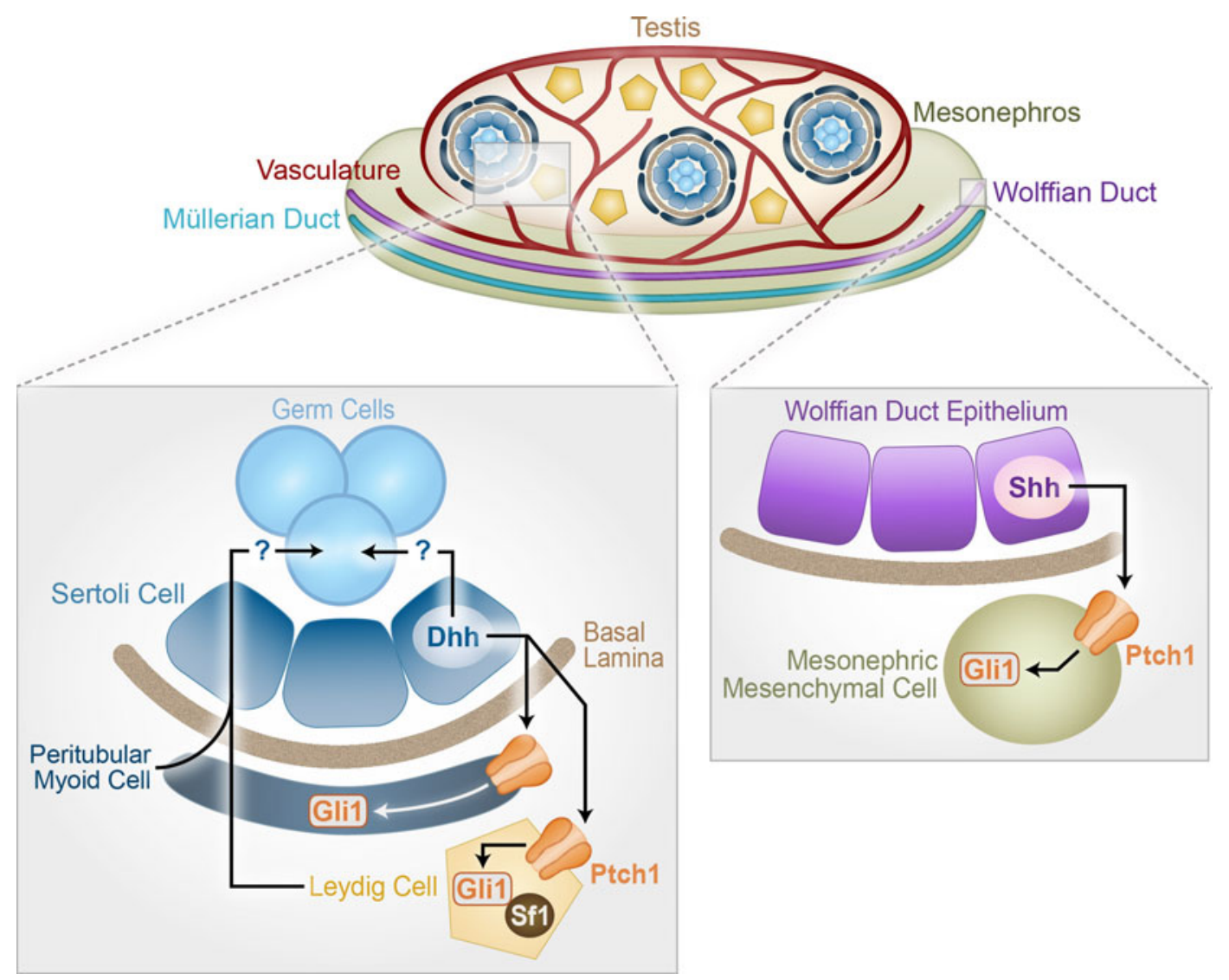

Fig. 1 Model of hedgehog action in the fetal male reproductive system. The fetal testis is composed of testis cords that encapsulate the developing germ cells and Sertoli cells, an extensive vascular network, and an interstitial compartment that contains the steroidogenic Leydig cells and peritubular myoid cells among other cell types. DHH produced by the Sertoli cells signals through PTCH1 and GLI1 in the Leydig and peritubular myoid cells to regulate their development and overall testis cord organization. Interestingly, despite the same hedgehog signaling components, these two cell types respond differently to the DHH ligand possibly due to the presence of SF1 in the Leydig cells. DHH signaling has also been shown to influence spermatogenesis; however, it is unclear if this occurs through a direct or indirect mechanism. Within the mesonephros, SHH is produced by the Wolffian duct epithelium and PTCH1 and GLI1 are expressed in the mesonephric mesenchyme. Together, these data indicate that the hedgehog signaling pathway mediates epithelial-mesenchymal communication during fetal male reproductive development 
McMahon 1995). This inactive state of the hedgehog signaling pathway is necessary, otherwise it triggers the ectopic appearance of fetal Leydig cells (Barsoum et al. 2009). However, after birth, all three Hedgehog ligands are detected (Russell et al. 2007). In the adult mouse ovary, both Dhh and $I h h$ are expressed, exhibiting localization in the granulosa cells of the antral follicles (Russell et al. 2007; Wijgerde et al. 2005). Components of the hedgehog signaling pathway, Ptch1, Smo, and Gli1, are localized mostly to the theca cell layer of the follicles and are also found at lower levels in the granulosa cells and other cell types within the ovarian stroma. Similar patterns of expression of the hedgehog signaling pathway were observed in both rat and bovine ovaries, suggesting that this pathway may be conserved in the ovary (Spicer et al. 2009). Loss of hedgehog signaling has not been reported to influence folliculogenesis (Wijgerde et al. 2005). Rather, ectopic activation of the hedgehog signaling pathway disrupts folliculogenesis. In in vitro culture of bovine theca cells, treatment with recombinant $\mathrm{SHH}$ increased their proliferation and androstenedione production (Spicer et al. 2009). Additionally, recombinant SHH treatment increased the proliferation and growth of follicles in vitro (Russell et al. 2007). However, genetic activation of the hedgehog signaling pathway in vivo inhibited follicle development, specifically the development of the theca cell population, resulting in female infertility (Ren et al. 2009). These observations highlight the importance of coordinate hedgehog action in the adult ovary, similar to the testis.

While there is no functional data that implicate the hedgehog signaling pathway in mouse ovarian development, much is known about its role in Drosophila. The Drosophila ovary develops from the anterior somatic cells that form terminal filaments to define each ovariole (Jemc 2011). At the same time, the cap cells appear and contribute to the germ stem cell niche or germarium. Within each ovariole, egg chambers form and contain the developing germ cells. The germ cells are encapsulated by a population of somatic cells, known as the escort cells, that are eventually replaced by follicle cells stalk cells, and polar cells as oogenesis progresses. Manipulation of the hedgehog signaling pathway in the egg chamber reveals that the development of the pre-follicle and pre-stalk cells as well as the positioning and development of the germ cells require a coordinated hedgehog signaling pathway (Besse et al. 2005, 2002; Forbes et al. 1996; Zhang and Kalderon 2000). Genetic models in mice will provide much needed information as to whether the role of the hedgehog signaling pathway in ovarian development is conserved among different species.

\section{Let us not ignore the reproductive tracts}

Following the establishment of the gonads, the reproductive tracts undergo dramatic remodeling and transform from a straight tubule into a convoluted and segmented structure. Although the components of the hedgehog signaling pathway are present in the tracts, their roles in tract development have not yet been identified. The Wolffian duct epithelium is the source of $S h h$, which is the only hedgehog ligand present in the mesonephros (Bitgood and McMahon 1995). Both Ptch1 and Gli1 are expressed in the mesonephric mesenchyme, implying an epithelial-mesenchymal crosstalk in this tissue (Fig. 1) (Barsoum and Yao 2011; Bitgood et al. 1996; Yao et al. 2002). Studies in Drosophila have implicated the hedgehog signaling pathway in reproductive tract development. Genital discs, the precursors of the reproductive tracts in Drosophila, are specified along the anterior posterior (A-P) axis (Gorfinkiel et al. 2003). The hedgehog signaling pathway defines these A-P borders as manipulating hedgehog levels in the different segments alters the resulting internal genitalia. Additionally, the hedgehog response is dependent on the anterior or posterior position of the responding cell with regards to the signaling cell (Gorfinkiel et al. 2003). These data imply that the hedgehog signaling pathway could potentially influence the development of the reproductive tracts and, combined with the expression data gathered from mice, suggests that this could be a conserved mechanism of reproductive tract development.

Epithelial-mesenchymal communication via the hedgehog signaling pathway seems to be maintained in the adult reproductive tract, similar to that of the fetal stage. In the adult male reproductive tract, the expression pattern of Shh and its downstream components remains similar to their fetal counterpart (Turner et al. 2006, 2004). In the adult female reproductive tract, Ihh is expressed in the uterine epithelium, 
whereas its effectors Ptch1, Gli1, and COUP-TFII are expressed in the uterine stroma (Matsumoto et al. 2002; Takamoto et al. 2002). Inhibition of Hedgehog signaling by cyclopamine treatment impaired sperm motility in the adult epididymis (Turner et al. 2006). These studies corroborate studies in the female that demonstrated that the hedgehog signaling pathway is necessary for female fertility as a key regulator of uterine function (Franco et al. 2010a; Kurihara et al. 2007; Lee et al. 2010, 2006; Takamoto et al. 2005). In addition, the hedgehog signaling pathway is necessary for oviductal coiling, uterine gland formation, and proper differentiation of the uterine epithelial and stromal compartments (Franco et al. 2010b; Migone et al. 2011). Thus, these studies describe a role for the hedgehog signaling pathway in epithelial-mesenchymal communication in the regulation of adult reproductive tract development and function, but the question remains as to its role in fetal reproductive tract development.

\section{In this case, it is not just what is on the inside that counts}

Establishment of the external genitalia is the final step of sexual differentiation and, not surprisingly, is also controlled by the hedgehog signaling pathway. In both male and female mouse embryos, the external genitalia start to develop as genital swellings around E10.5, which eventually form the bipotential genital tubercle (Perriton et al. 2002). The genital tubercle then undergoes further outgrowth, accompanied by mesenchymal condensations. At E16.5, the first sexspecific morphological differences in the external genitalia are evident as the male organ is more pronounced than its female counterpart (Haraguchi et al. 2000; Miyagawa et al. 2011). The hedgehog ligand, $S h h$, is expressed in the urethral epithelium, whereas its downstream signaling components, Ptch1, Gli1, Gli2, and Gli3, are expressed in the mesenchyme of the mouse external genitalia, revealing a similar pattern of epithelial-mesenchymal crosstalk as the other reproductive organs (Haraguchi et al. 2001, 2000). $\mathrm{Shh}^{-/-}$mice display small residual external genitalia structures in both males and females, implicating the hedgehog signaling pathway in the initiation of genital tubercle outgrowth (Haraguchi et al. 2001; Perriton et al. 2002). In addition to its role in the initiation of genital tubercle outgrowth, Shh is also essential for early urethral plate formation and subsequent growth and patterning of the penis and closure of the urethral tube (Haraguchi et al. 2001; Seifert et al. 2009). Ablation of Shh resulted in hypospadias or abnormal closure of the urethra. Strikingly, the duration of Shh ablation impacted the overall length of the genitalia in a manner reminiscent of the limb, indicating that both the initiation and duration of the hedgehog signal affects external genitalia development. The mechanism of SHH action in the external genitalia is likely through its inhibition of cell death and promotion of cell proliferation (Haraguchi et al. 2001; Perriton et al. 2002; Seifert et al. 2009, 2010).

The role of SHH was further validated by manipulating downstream components of the hedgehog signaling pathway in the external genitalia. When Smo was ablated in the genital tubercle ectoderm, male mice developed hypospadias similar to the Shh knockout model; however, ablation of Smo in Shh-expressing cells had no impact on genital development (Seifert et al. 2009). Taking an alternative approach, Miyagawa et al. (2011) produced mouse models where SMO was ectopically activated in the female genital tubercle. While a partial masculinization occurred, a complete sex reversal of the female external genitalia to male was not observed, indicating that the hedgehog signaling pathway alone is not sufficient to induce male-specific patterning of the external genitalia. Downstream of $\mathrm{SHH}$ and SMO, GLI2 is the predominant intracellular regulator in the mesenchyme of the genital tubercle (Haraguchi et al. 2001; Miyagawa et al. 2011). Interestingly, while $\mathrm{Gli}^{-/-}$mice develop normally, when three copies of Gli2 or Gli3 are inactivated $\left(\right.$ Gli2 $^{+/-}$Gli3 $^{-/}$, Gli2 $^{-/}$Gli3 $\left.^{+/-}\right)$, the resulting genital phenotypes are more severe than either knockout alone (Miyagawa et al. 2011). An additional striking feature of the $\mathrm{Gli}^{-/-}$mice is that the female genital phenotype is much less severe than that of the male mice, suggesting that the hedgehog signaling pathway influences the sexually dimorphic development of the external genitalia. Interestingly, Drosophila with ectopic hedgehog expression in the genital discs develops duplicate external genitalia (Gorfinkiel et al. 2003). Thus, the hedgehog signaling pathway represents a conserved mechanism of external genitalia development. 


\section{Summary and conclusion}

The dynamic nature of sexual differentiation presents a robust system for the study of the mechanisms by which cellular crosstalk influences developmental events. Based on evidence from in vivo model systems and in vitro organ culture, the hedgehog signaling pathway is clearly involved in cell fate determination and cell-cell communication in the process. Rather than controlling the primary sex determination of the gonads, the hedgehog signaling pathway plays critical roles in the dimorphic establishment of the gonads, reproductive tract, and external genitalia. The primary mechanism by which the hedgehog signaling pathway acts is through epithelial-mesenchymal crosstalk, with the epithelium being the source of ligand and surrounding mesenchyme as the target. This crosstalk is apparent in both the testis and external genitalia. The expression of hedgehog components in the ovary and the Wolffian duct suggests the presence of a similar mechanism. While much has been uncovered about the involvement of the hedgehog signaling pathway in sexual differentiation, many questions remain to be addressed. For instance, how does the hedgehog signaling pathway induce different cellular responses in the precursors of fetal Leydig and peritubular myoid cells? Do hedgehog ligands from other sources, such as the mesonephros, contribute to overall testis development and influence reproductive tract development? How does the hedgehog signaling pathway influence germ cell differentiation in the testis and ovary? Addressing these questions will provide a better understanding of not only sexual differentiation but also how the hedgehog signaling pathway mediates cellular response in general.

Disorders of sexual development affect 1 in 4,5005,000 live births and are a major health issue (Warne and Raza 2008). One class of these disorders is defined by abnormal development of the reproductive system, such as gonadal dysgenesis and hypospadias. The etiology of these disorders is largely undefined and only $10-15 \%$ of cases have been linked to specific genetic mutations (Canto et al. 2004; Das et al. 2011). Mutations in $D H H$ have been identified in patients with gonadal dysgenesis (Canto et al. 2004, 2005; Das et al. 2011; Umehara et al. 2000). These patients display variable reproductive and neuropathological phenotypes that resemble the $D h h^{-1-}$ mouse model.
Mutations in the other members of the hedgehog signaling pathway have not been linked to disorders of sexual development, likely due to the importance of these genes in embryonic survival (Cohen 2003; Varjosalo and Taipale 2008). However, future studies may identify the hedgehog signaling pathway as a genetic modifier(s), which along with other mutations acting in a polygenic or gene-dosage-dependent manner, facilitate disorders of sexual development in humans.

In conclusion, through the use of model systems, the hedgehog signaling pathway has been shown to be a conserved mechanism through which the reproductive system develops. A pattern has emerged in which the hedgehog signal emanates from the epithelium and signals through its downstream components in the mesenchyme. As further information is gathered as to the mechanisms by which the hedgehog signaling pathway regulates this crosstalk, its potential impact on disorders of sexual development will become apparent eventually leading to treatments for these disorders.

Acknowledgments We would like to thank Gen Yamada and the members of the Yao laboratory for helpful comments on the manuscript. We also thank Sue Edelstein from the NIEHS graphics department for assistance with figures. H.L.F. and H.H-C.Y. are both supported by the National Institute of Health Intramural Research Fund.

\section{References}

Arur S, Ohmachi M, Berkseth M, Nayak S, Hansen D, Zarkower D, Schedl T (2011) MPK-1 ERK controls membrane organization in C. elegans oogenesis via a sex-determination module. Dev Cell 20:677-688

Barsoum IB, Yao HHC (2009) Fetal Leydig cells: progenitor cell maintenance and differentiation. J Androl 31:11-15

Barsoum I, Yao HHC (2011) Redundant and differential roles of transcription factors Gli1 and Gli2 in the development of mouse fetal Leydig cells. Biol Reprod 84:894-899

Barsoum IB, Bingham NC, Parker KL, Jorgensen JS, Yao HHC (2009) Activation of the hedgehog pathway in the mouse fetal ovary leads to ectopic appearance of fetal Leydig cells and female pseudohermaphroditism. Dev Biol 329:96-103

Besse F, Busson D, Pret AM (2002) Fused-dependent hedgehog signal transduction is required for somatic cell differentiation during Drosophila egg chamber formation. Development 129:4111-4124

Besse F, Busson D, Pret A-M (2005) Hedgehog signaling controls Soma-Germen interactions during Drosophila ovarian morphogenesis. Dev Dyn 234:422-431 
Bitgood MJ, McMahon AP (1995) Hedgehog and Bmp genes are coexpressed at many diverse sites of cellcell interaction in the mouse embryo. Dev Biol 172:126-138

Bitgood MJ, Shen L, McMahon AP (1996) Sertoli cell signaling by Desert hedgehog regulates the male germline. Curr Biol 6:298-304

Canto P, Soderlund D, Reyes E, Mendez JP (2004) Mutations in the desert hedgehog (DHH) gene in patients with 46, $\mathrm{XY}$ complete pure gonadal dysgenesis. J Clin Endocrinol Metab 89:4480-4483

Canto P, Vilchis F, Soderlund D, Reyes E, Mendez JP (2005) A heterozygous mutation in the desert hedgehog gene in patients with mixed gonadal dysgenesis. Mol Hum Reprod 11:833-836

Clark AM, Garland KK, Russell LD (2000) Desert hedgehog (Dhh) gene is required in the mouse testis for formation of adult-type Leydig cells and normal development of peritubular cells and seminiferous tubules. Biol Reprod 63:1825-1838

Cohen MM Jr (2003) The hedgehog signaling network. Am J Med Genet A 123A:5-28

Combes AN, Wilhelm D, Davidson T, Dejana E, Harley V, Sinclair A, Koopman P (2009) Endothelial cell migration directs testis cord formation. Dev Biol 326:112-120

Cool J, DeFalco TJ, Capel B (2011) Vascular-mesenchymal cross-talk through Vegf and Pdgf drives organ patterning. Proc Natl Acad Sci U S A 108:167-172

Das DK, Sanghavi D, Gawde H, Idicula-Thomas S, Vasudevan L (2011) Novel homozygous mutations in Desert Hedgehog gene in patients with 46, XY complete gonadal dysgenesis and prediction of its structural and functional implications by computational methods. Eur J Med Genet 54:e529-e534

Deshpande G, Swanhart L, Chiang P, Schedl P (2001) Hedgehog signaling in germ cell migration. Cell 106:759-769

Driver EC, Pryor SP, Hill P, Turner J, Ruther U, Biesecker LG, Griffith AJ, Kelley MW (2008) Hedgehog signaling regulates sensory cell formation and auditory function in mice and humans. J Neurosci 28:7350-7358

Fan J, Akabane H, Zheng X, Zhou X, Zhang L, Liu Q, Zhang YL, Yang J, Zhu GZ (2007) Male germ cell-specific expression of a novel Patched-domain containing gene Ptchd3. Biochem Biophys Res Commun 363:757-761

Forbes AJ, Lin H, Ingham PW, Spralding AC (1996) Hedgehog is required for the proliferation and specification of ovarian somatic cells prior to egg chamber formation in Drosophila. Development 122:1125-1135

Franco HL, Lee KY, Broaddus RR, White LD, Lanske B, Lydon JP, Jeong JW, DeMayo FJ (2010a) Ablation of Indian hedgehog in the murine uterus results in decreased cell cycle progression, aberrant epidermal growth factor signaling, and increased estrogen signaling. Biol Reprod 82:783-790

Franco HL, Lee KY, Rubel CA, Creighton CJ, White LD, Broaddus RR, Lewis MT, Lydon JP, Jeong JW, DeMayo FJ (2010b) Constitutive activation of smoothened leads to female infertility and altered uterine differentiation in the mouse. Biol Reprod 82:991-999
Gorfinkiel N, Sanchez L, Guerrero I (2003) Development of the Drosophila genital disc requires interactions between its segmental primordia. Development 130:295-305

Haraguchi R et al (2000) Molecular analysis of external genitalia formation: the role of fibroblast growth factor (Fgf) genes during genital tubercle formation. Development 127:2471-2479

Haraguchi R, Mo R, Hui CC, Motoyama J, Makino S, Shiroishi T, Gaffield W, Yamada G (2001) Unique functios of Sonic hedgehog signalig during external genitalia development. Development 128:4241-4250

Ingham PW, Nakano Y, Seger C (2011) Mechanisms and functions of Hedgehog signalling across the metazoa. Nat Rev Genet 12:393-406

Jeanes A, Wilhelm D, Wilson MJ, Bowles J, McClive PJ, Sinclair AH, Koopman P (2005) Evaluation of candidate markers for the peritubular myoid cell lineage in the developing mouse testis. Reproduction 130:509516

Jemc JC (2011) Somatic gonadal cells: the supporting cast for the germline. Genesis 49:753-775

Kawai Y, Noguchi J, Akiyama K, Takeno Y, Fujiwara Y, Kajita S, Tsuji T, Kikuchi K, Kaneko H, Kunieda T (2011) A missense mutation of the Dhh gene is associated with male pseudohermaphroditic rats showing impaired Leydig cell development. Reproduction 141:217225

Kobayashi A, Behringer RR (2003) Developmental genetics of the female reproductive tract in mammals. Nat Rev Genet 4:969-980

Koopman P, Gubbay J, Vivian N, Goodfellow P, Lovell-Badge R (1991) Male development of chromosomally female mice transgenic for Sry. Nature 351:117-121

Kroft TL, Patterson J, Won Yoon J, Doglio L, Walterhouse DO, Iannaccone PM, Goldberg E (2001) GLI1 localization in the germinal epithelial cells alternates between cytoplasm and nucleus: upregulation in transgenic mice blocks spermatogenesis in pachytene. Biol Reprod 65:1663-1671

Kurihara I, Lee DK, Petit FG, Jeong J, Lee K, Lydon JP, DeMayo FJ, Tsai MJ, Tsai SY (2007) COUP-TFII mediates progesterone regulation of uterine implantation by controlling ER activity. PLoS Genet 3:e102

Kuwabara PE, Lee MH, Schedl T, Jefferis GS (2000) A C. elegans patched gene, ptc-1, functions in germ-line cytokinesis. Genes Dev 14:1933-1944

Lee $\mathrm{K}$ et al (2006) Indian hedgehog is a major mediator of progesterone signaling in the mouse uterus. Nat Genet 38:1204-1209

Lee DK, Kurihara I, Jeong JW, Lydon JP, DeMayo FJ, Tsai MJ, Tsai SY (2010) Suppression of ERalpha activity by COUP-TFII is essential for successful implantation and decidualization. Mol Endocrinol 24:930-940

Lee FY, Faivre EJ, Suzawa M, Lontok E, Ebert D, Cai F, Belsham DD, Ingraham HA (2011) Eliminating SF-1 (NR5A1) sumoylation in vivo results in ectopic hedgehog signaling and disruption of endocrine development. Dev Cell 21:315-327

Lin FJ, Qin J, Tang K, Tsai SY, Tsai MJ (2011) Coup d'Etat: an orphan takes control. Endocr Rev 32:404-421 
Lovell-Badge R, Robertson E (1990) XY female mice resulting from a heritable mutation in the primary testis-determining gene, Tdy. Development 109:635-646

Matsumoto H, Zhao X, Das SK, Hogan BL, Dey SK (2002) Indian hedgehog as a progesterone-responsive factor mediating epithelial-mesenchymal interactions in the mouse uterus. Dev Biol 245:280-290

Mich JK, Blaser H, Thomas NA, Firestone AJ, Yelon D, Raz E, Chen JK (2009) Germ cell migration in zebrafish is cyclopamine-sensitive but Smoothened-independent. Dev Biol 328:342-354

Migone FF, Ren Y, Cowan RG, Harman RM, Nikitin AY, Quirk SM (2011) Dominant activation of the hedgehog signaling pathway alters development of the female reproductive tract. Genesis. doi:10.1002/dvg.20786

Miyagawa $\mathrm{S}$ et al (2011) The role of sonic hedgehog-gli2 pathway in the masculinization of external genitalia. Endocrinology 152:2894-2903

Morales CR, Fox A, El-Alfy M, Ni X, Argraves WS (2009) Expression of Patched-1 and Smoothened in testicular meiotic and post-meiotic cells. Microsc Res Tech 72:809815

Nusslein-Volhard C, Wieschaus E (1980) Mutations affecting segment number and polarity in Drosophila. Nature 287:795-801

Park SY, Tong M, Jameson JL (2007) Distinct roles for steroidogenic factor 1 and desert hedgehog pathways in fetal and adult Leydig cell development. Endocrinology 148:3704-3710

Perriton CL, Powles N, Chiang C, Maconochie MK, Cohn MJ (2002) Sonic hedgehog signaling from the urethral epithelium controls external genitalia development. Dev Biol 247:26-46

Pierucci-Alves F, Clark AM, Russell LD (2001) A developmental study of the Desert hedgehog-null mouse testis. Biol Reprod 65:1392-1402

Qin J, Tsai MJ, Tsai SY (2008) Essential roles of COUP-TFII in Leydig cell differentiation and male fertility. PLoS One 3:e3285

Ren Y, Cowan RG, Harman RM, Quirk SM (2009) Dominant activation of the hedgehog signaling pathway in the ovary alters theca development and prevents ovulation. Mol Endocrinol 23:711-723

Renault AD, Ricardo S, Kunwar PS, Santos A, Starz-Gaiano M, Stein JA, Lehmann R (2009) Hedgehog does not guide migrating Drosophila germ cells. Dev Biol 328:355-362

Russell MC, Cowan RG, Harman RM, Walker AL, Quirk SM (2007) The hedgehog signaling pathway in the mouse ovary. Biol Reprod 77:226-236

Sato T, Ogata J, Niki Y (2010) BMP and Hh signaling affects primordial germ cell division in Drosophila. Zoological Science 27:804-810

Seifert AW, Bouldin CM, Choi KS, Harfe BD, Cohn MJ (2009) Multiphasic and tissue-specific roles of sonic hedgehog in cloacal septation and external genitalia development. Development 136:3949-3957

Seifert AW, Zheng Z, Ormerod BK, Cohn MJ (2010) Sonic hedgehog controls growth of external genitalia by regulating cell cycle kinetics. Nature Communications $1: 23$
Spicer LJ, Sudo S, Aad PY, Wang LS, Chun SY, Ben-Shlomo I, Klein C, Hsueh AJ (2009) The hedgehog-patched signaling pathway and function in the mammalian ovary: a novel role for hedgehog proteins in stimulating proliferation and steroidogenesis of theca cells. Reproduction 138:329-339

Szczepny A, Hime GR, Loveland KL (2006) Expression of hedgehog signalling components in adult mouse testis. Dev Dyn 235:3063-3070

Szczepny A, Hogarth CA, Young J, Loveland KL (2008) Identification of hedgehog signaling outcomes in mouse testis development using a hanging drop-culture system. Biol Reprod 80:258-263

Takamoto N, Zhao B, Tsai SY, DeMayo FJ (2002) Identification of Indian hedgehog as a progesteroneresponsive gene in the murine uterus. Mol Endocrinol $16: 2338-2348$

Takamoto N, Kurihara I, Lee K, Demayo FJ, Tsai MJ, Tsai SY (2005) Haploinsufficiency of chicken ovalbumin upstream promoter transcription factor II in female reproduction. Mol Endocrinol 19:2299-2308

Tung PS, Skinner MK, Fritz IB (1984) Cooperativity between sertoli cells and peritubular myoid cells in the formation of the basal lamina in the seminiferous tubule. Ann N Y Acad Sci 438:435-446

Turner TT, Bomgardner D, Jacobs JP (2004) Sonic hedgehog pathway genes are expressed and transcribed in the adult mouse epididymis. J Androl 25:514-522

Turner TT, Bang HJ, Attipoe SA, Johnston DS, Tomsig JL (2006) Sonic hedgehog pathway inhibition alters epididymal function as assessed by the development of sperm motility. J Androl 27:225-232

Umehara F, Tate G, Itoh K, Yamaguchi N, Douchi T, Mitsuya T, Osame M (2000) A novel mutation of desert hedgehog in a patient with 46, XY partial gonadal dysgenesis accompanied by minifascicular neuropathy. Am J Hum Genet 67:1302-1305

Varjosalo M, Taipale J (2008) Hedgehog: functions and mechanisms. Genes Dev 22:2454-2472

Vied C, Horabin JI (2001) The sex determination master switch, Sex-lethal, responds to Hedgehog signaling in the Drosophila germline. Development 128:2649-2660

Walterhouse DO, Lamm ML, Villavicencio E, Iannaccone PM (2003) Emerging roles for hedgehog-patched-Gli signal transduction in reproduction. Biol Reprod 69:814

Warne GL, Raza J (2008) Disorders of sex development (DSDs), their presentation and management in different cultures. Rev Endocr Metab Disord 9:227-236

Welshons WJ, Russell LB (1959) The Y-chromosome as the bearer of male determining factors in the mouse. Proc Natl Acad Sci U S A 45:560-566

Wijgerde M, Ooms M, Hoogerbrugge JW, Grootegoed JA (2005) Hedgehog signaling in mouse ovary: Indian hedgehog and desert hedgehog from granulosa cells induce target gene expression in developing theca cells. Endocrinology 146:3558-3566

Wilson CW, Chuang PT (2010) Mechanism and evolution of cytosolic Hedgehog signal transduction. Development 137:2079-2094 
Xia L et al (2010) The Fused/Smurf complex controls the fate of Drosophila germline stem cells by generating a gradient BMP response. Cell 143:978-990

Yao HH, Capel B (2002) Disruption of testis cords by cyclopamine or forskolin reveals independent cellular pathways in testis organogenesis. Dev Biol 246:356-365
Yao HH, Whoriskey W, Capel B (2002) Desert Hedgehog/ Patched 1 signaling specifies fetal Leydig cell fate in testis organogenesis. Genes Dev 16:1433-1440

Zhang Y, Kalderon D (2000) Regulation of cell proliferation and patterning in Drosophila oogenesis by Hedgehog signaling. Development 127:2165-2176 